\title{
Design principles for particle plasmon enhanced solar cells
}

\author{
K. R. Catchpole ${ }^{\text {a) }}$ and A. Polman ${ }^{\text {b) }}$ \\ Center for Nanophotonics, FOM Institute for Atomic and Molecular Physics (AMOLF), Kruislaan 407, \\ 1098 SJ Amsterdam, The Netherlands
}

(Received 1 August 2008; accepted 20 October 2008; published online 14 November 2008)

\begin{abstract}
We develop fundamental design principles for increasing the efficiency of solar cells using light trapping by scattering from metal nanoparticles. We show that cylindrical and hemispherical particles lead to much higher path length enhancements than spherical particles, due to enhanced near-field coupling, and that the path length enhancement for an electric point dipole is even higher than the Lambertian value. Silver particles give much higher path length enhancements than gold particles. The scattering cross section of the particles is very sensitive to the thickness of a spacer layer at the substrate, which provides additional tunability in the design of particle arrays. () 2008 American Institute of Physics. [DOI: 10.1063/1.3021072]
\end{abstract}

Silicon solar cells require light trapping for high performance because of weak absorption at long wavelengths due to the indirect band gap. For wafer-based cells, inverted or upright pyramids can be used for light trapping, which have a typical feature size of around $10 \mu \mathrm{m} .{ }^{1}$ However, for thinfilm solar cells, which may have a thickness of only 1-2 $\mu \mathrm{m}$, such textures are not suitable. While submicron surface textures can be used to achieve light trapping, these also increase the surface area and hence increase minority carrier recombination in the surface and junction regions, which is particularly detrimental for the efficiency of thinfilm cells. A way of achieving light trapping in thin-film solar cells that has emerged recently is the use of scattering from metal nanoparticles. ${ }^{2-5}$ This approach has led to an increase in photocurrent in $1.25 \mu \mathrm{m}$ thick silicon-on-insulator solar cells of up to a factor of 16 at long wavelengths and an integrated increase in photocurrent across the solar spectrum of over $30 \%{ }^{2}$ By reciprocity, the use of metal nanoparticles can also increase the external quantum efficiency from lightemitting diodes. A nearly 12-fold increase in electroluminescence for thin silicon-on-insulator light-emitting diodes has been reported in this way. ${ }^{2,6}$ In the latter reports the metal nanoparticles were formed by evaporation of a thin metal film followed by annealing, which leads to randomly distributed particles with a flattened roughly hemispherical shape. Enhancements in photocurrent have also been reported using spherical colloidal nanoparticles deposited onto silicon solar cells. ${ }^{3}$

Despite these initial studies, no systematic research has been performed to investigate the optimum particle parameters and the optimum light trapping that can be achieved using scattering from metal nanoparticles. In this paper we investigate light scattering from a single $\mathrm{Ag}$ or Au particle, varying the shape, size, particle material, and dielectric environment, in order to determine some fundamental design principles for particle plasmon enhanced light trapping. The work is done with solar cell applications in mind, but the results are relevant for light-emitting diodes as well. We show that cylindrical and hemispherical particles are much

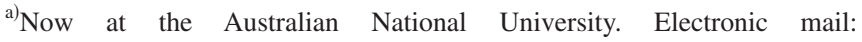
kylie.catchpole@anu.edu.au.

b)Electronic mail: polman@amolf.nl.
}

more effective than spherical particles at scattering light into a high-index substrate and keeping the light trapped within the substrate due to enhanced near-field coupling. We also show that the scattering cross section of the particles on the substrate is relatively large and can be increased further by increasing the distance of the particles from the substrate.

A measure of the effectiveness of light trapping is the path length enhancement that weakly absorbed light experiences compared to the path length for a single pass across the device. To estimate the potential path length enhancements we use finite-difference time-domain calculations ${ }^{7}$ to model the scattering from $\mathrm{Ag}$ and $\mathrm{Au}$ particles under normal incidence using perfectly matched layer boundary conditions. The metal particles are separated from a semi-infinite Si substrate by a thin silicon dioxide or silicon nitride layer. Such layers are present in solar cells for surface passivation. The dielectric functions are modeled using a Drude model for $\mathrm{Ag}$ and a Drude-Lorentz model for $\mathrm{Si}$ and Au. A mesh size of $0.5-1 \mathrm{~nm}$ in the vicinity of the metal particle was used. The total field and scattered field around the particle are calculated as a function of wavelength. The scattered power is calculated by integrating the Poynting vector of the scattered field over a box surrounding the particle. This can be separated into the power scattered into the air and the power scattered into the substrate by integrating over the relevant parts of the simulation box. The normalized scattering cross section, $Q_{\text {scat }}$, is then calculated by dividing the scattered power by the incident intensity and by the geometrical cross section, i.e., the area of the particle projected on the substrate. The power absorbed by the particle is calculated by integrating the Poynting vector for the total field over the surface of a box enclosing the particle. The absorbed power is then divided by the incident intensity and by the geometrical cross section to obtain the normalized absorption cross section $Q_{\text {abs }}$.

The most important factor determining the path length enhancement of a scattering light-trapping structure is the fraction of light that is scattered into the substrate, $f_{\text {subs }}$, defined as the power scattered into the substrate divided by the total scattered power. Because the path length enhancement depends on multiple scattering events, the fraction of light lost into the air at each surface scattering event must be small. Figure 1 shows $f_{\text {subs }}$ calculated for Ag particles on a 


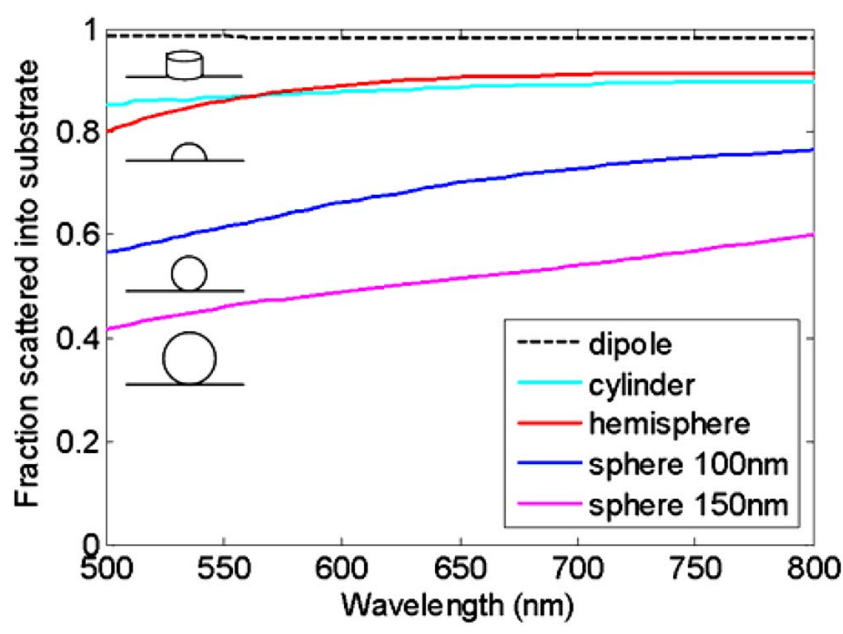

FIG. 1. (Color online) Fraction of light scattered into the substrate, $f_{\text {subs }}$, for Ag particles on a $10 \mathrm{~nm}$ thick $\mathrm{SiO}_{2}$ underlayer on $\mathrm{Si}$ : a cylinder with diameter $d=100 \mathrm{~nm}$ and height $h=50 \mathrm{~nm}$; a $100 \mathrm{~nm}$ diameter hemisphere; a $100 \mathrm{~nm}$ diameter sphere; and a $150 \mathrm{~nm}$ diameter sphere. Also plotted is $f_{\text {subs }}$ for a parallel electric dipole that is $10 \mathrm{~nm}$ from a Si substrate.

$10 \mathrm{~nm}$ thick $\mathrm{SiO}_{2}$ underlayer on a semi-infinite $\mathrm{Si}$ substrate with a range of different shapes: a cylinder with diameter $d=100 \mathrm{~nm}$ and height $h=50 \mathrm{~nm}$; a $100 \mathrm{~nm}$ diameter hemisphere; a $100 \mathrm{~nm}$ diameter sphere; and a $150 \mathrm{~nm}$ diameter sphere. A calculation of $f_{\text {subs }}$ for a parallel polarized electric point dipole at a distance $t=10 \mathrm{~nm}$ from the $\mathrm{Si}$ substrate (using the method of Mertz ${ }^{8}$ ) is also shown in Fig. 1. As can be seen in the figure, for the entire wavelength range the fraction scattered into the substrate is much higher for the cylinder and hemisphere than that for the spheres. For spheres, the value of $f_{\text {subs }}$ decreases significantly as the sphere diameter is increased. For 100 and $150 \mathrm{~nm}$ diameter cylinders $f_{\text {subs }}$ does not vary significantly with the particle diameter (not shown). We attribute the high fraction of light scattered into the substrate for the cylinder and the hemisphere to the fact that the average spacing to the substrate is smaller for these geometries than for spheres. This allows efficient coupling of the part of the scattered light that has high in-plane wave vector and is evanescent in air but can propagate in silicon.

The other factor affecting the path length enhancement is the angle of propagation of light through the device. The effect of oblique propagation can be described by a factor $d_{\mathrm{av}}$, which is the ratio of the path length across a single pass of the device to the device thickness, averaged over the angular distribution of the light. The inset of Fig. 2 shows an angular plot of the relative power scattered per unit solid angle for an electric point dipole parallel to and separated by $10 \mathrm{~nm}$ from a silicon surface. The cosine dependent Lambertian scattering distribution, characteristic of surfaces with wavelength-scale surface texturing, is shown for reference. The scattering distribution for the dipole shows peaks above the critical angle in silicon and overall the light is scattered closer to the normal than for a Lambertian scatterer. Values of $d_{\mathrm{av}}$ are determined by performing a weighted integral of $1 / \cos (\theta)$ over the angular distribution of the scattered power, with $\theta$ as the angle with the normal. We find $d_{\mathrm{av}}=1.5$ for an electric point dipole parallel to and $10 \mathrm{~nm}$ from a Si surface, compared to a value of 2 for Lambertian scattering. $d_{\mathrm{av}}$ decreases as the distance between the dipole and the substrate is increased. For a dipole with $f_{\text {subs }}=90 \%$ (spaced at a dis-

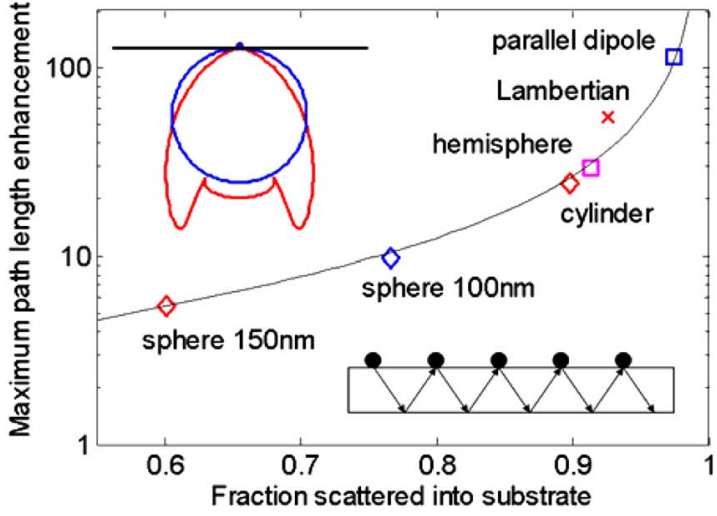

FIG. 2. (Color online) Maximum path length enhancement for the same geometries as in Fig. 1 at a wavelength of $800 \mathrm{~nm}$. Absorption within the particles is neglected for these calculations, and an ideal rear reflector is assumed. The line is a guide for the eyes. Insets: (top-left) angular distribution of scattered power for a parallel electric dipole that is $10 \mathrm{~nm}$ above a Si surface and Lambertian scatterer; (bottom-right) geometry considered for calculating the path length enhancement.

tance $t=45 \mathrm{~nm}$ ) the value of $d_{\mathrm{av}}$ is 1.25 , whereas for a dipole with $f_{\text {subs }}=77 \%(t=73 \mathrm{~nm})$ the value of $d_{\mathrm{av}}$ is 1.15 . The decrease in the fraction scattered into the substrate for larger distance $t$ is associated with a decrease in the average angle of propagation because of the decrease in coupling of wavevectors with high in-plane momentum. For the particles studied here, we make the approximation that $d_{\mathrm{av}}$ equals the value for a horizontal electric dipole with the corresponding value of $f_{\text {subs. }}$.

Once $f_{\text {subs }}$ and $d_{\mathrm{av}}$ are known, the path length enhancement can be determined. By calculating the path length of the light coupled out through the front surface after each reflection from the rear, a geometric series for the average path length can be constructed, leading to a maximum path length enhancement and hence absorptance enhancement for weakly absorbed light of approximately ${ }^{9,10} 2 d_{\text {av }} /\left(1-f_{\text {subs }}\right)$. The factor of 2 is because an ideal rear reflector is assumed. For these calculations as an initial approximation we assume that $f_{\text {subs }}$ is independent of the angle of the incident light. Figure 2 shows the maximum path length enhancements obtained in this way plotted versus fraction scattered into the substrate for the particle geometries studied in Fig. 1 at a wavelength of $800 \mathrm{~nm}$. The Lambertian case, with $f_{\text {subs }}=1$ $-1 / n^{2}$ and a maximum path length enhancement of $4 n^{2}$ (where $n$ is the refractive index of the substrate), ${ }^{9}$ is also shown. We can see that the path length enhancement for a parallel electric point dipole is considerably higher than the Lambertian value. The value of $f_{\text {subs }}$ is $97.5 \%$ for a horizontal electric dipole located $10 \mathrm{~nm}$ above a silicon surface. Using $d_{\mathrm{av}}=1.5$ this leads to an overall path length enhancement as large as 114. For comparison, the Lambertian value of $4 n^{2}$ equals 54 . We can also see that the difference in $f_{\text {subs }}$ for the different particle geometries shown in Fig. 1 leads to large differences in path length enhancement. The path length enhancement for a $100 \mathrm{~nm}$ diameter hemisphere is 28 , while the path length enhancement for a $150 \mathrm{~nm}$ diameter sphere is only 5 .

Next, we evaluate the scattering and absorption cross sections for different geometries for $\mathrm{Ag}$ and Au. Figure 3 shows the total normalized scattering cross section, the cross section for light scattered into the substrate, and the absorption cross section for hemispherical $\mathrm{Ag}$ and $\mathrm{Au}$ particles on a 


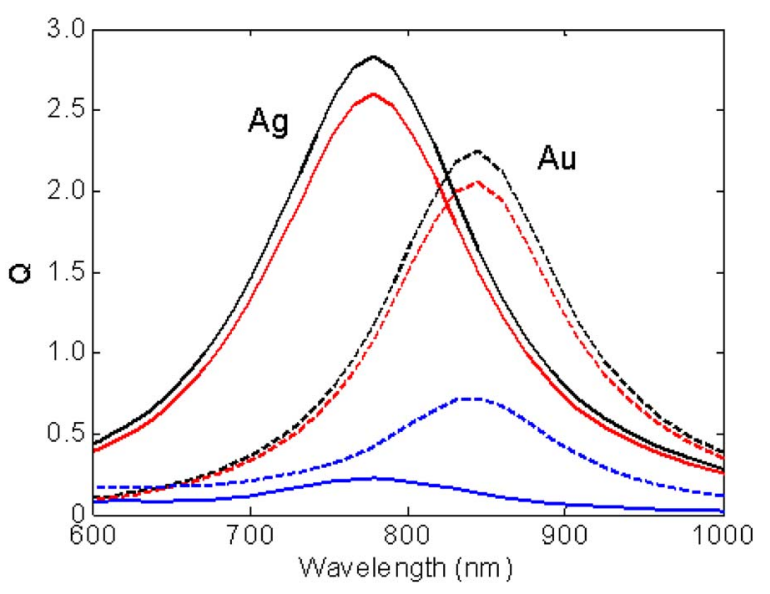

FIG. 3. (Color online) Total scattering cross section (black lines), cross section for light scattered into the substrate (red lines), and absorption cross section (blue lines), all normalized to geometrical cross section, for $100 \mathrm{~nm}$ diameter hemispherical particles of $\mathrm{Ag}$ (solid lines) and $\mathrm{Au}$ (dashed lines) on a $10 \mathrm{~nm}$ thick $\mathrm{Si}_{3} \mathrm{~N}_{4}$ underlayer on $\mathrm{Si}$.

$10 \mathrm{~nm}$ thick $\mathrm{Si}_{3} \mathrm{~N}_{4}(n=2)$ underlayer on a $\mathrm{Si}$ substrate. As can be seen, a very large fraction of the light is scattered into the substrate, in agreement with Fig. 1, at an effective crosssection well in excess of the particle area $(Q>1)$. The $\mathrm{Au}$ particle is considerably more absorbing than the $\mathrm{Ag}$ particle. We can calculate an effective maximum path length enhancement in the presence of absorption by using a corrected value for $f_{\text {subs }}$, equal to the power scattered into the substrate divided by the sum of the scattered power and the absorbed power. The maximum path length enhancement at the surface plasmon resonance for the $\mathrm{Ag}$ particle is then 17, while for the Au particle it is only 8 . The surface plasmon resonance wavelengths for the geometries in Fig. 3 are $770 \mathrm{~nm}$ for $\mathrm{Ag}$ and $840 \mathrm{~nm}$ for $\mathrm{Au}$, which are significantly redshifted compared to the values in air ( $350 \mathrm{~nm}$ for $\mathrm{Ag}$ and $480 \mathrm{~nm}$ for $\mathrm{Au}$, respectively). The fact that the resonance wavelengths for $\mathrm{Ag}$ and $\mathrm{Au}$ are so close is due to the similarity in the real part of the dielectric function of $\mathrm{Ag}$ and $\mathrm{Au}$ in the nearinfrared spectral range. Thus, it is preferable to use Ag particles and, by engineering the dielectric environment, shift the resonance to the desired wavelength range.

One way to further tune the surface plasmon resonance is by using a dielectric coating above and/or below the metal particle. ${ }^{11}$ Figure 4 shows the normalized total scattering cross section and cross section for light scattered into the substrate as a function of wavelength for $\mathrm{Ag}$ cylinders on $\mathrm{Si}_{3} \mathrm{~N}_{4}$ underlayers with thicknesses of 10 and $30 \mathrm{~nm}$, covered with a $30 \mathrm{~nm}$ thick $\mathrm{Si}_{3} \mathrm{~N}_{4}$ overlayer. For the $30 \mathrm{~nm}$ spacing $Q$ is as large as 10 on resonance, implying that a typical particle coverage of only $10 \%$ is required to fully scatter the incident power. As can be seen, increasing the underlayer thickness shifts the dipolar resonance from 880 to $780 \mathrm{~nm}$, due to reduced coupling of the plasmonic near field to the high-index Si substrate. A narrower peak near $600 \mathrm{~nm}$ due to quadrupole excitation is also observed. Hägglund et al. ${ }^{4}$ showed that the quadrupole mode couples much less effectively to the substrate than the dipole mode. From Fig. 4, we can see that decreasing the thickness of the underlying layer increases the fraction of light that is scattered into the substrate but decreases the scattering cross section of the particle. This is

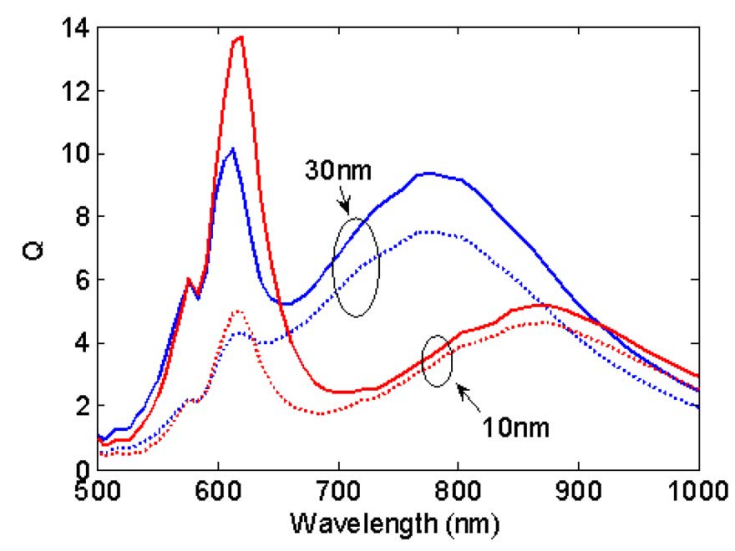

FIG. 4. (Color online) The total scattering cross section (solid lines) and scattering cross section of light scattered into the substrate (dashed lines), both normalized to geometrical cross section, for $\mathrm{Ag}$ cylinders on $\mathrm{Si}_{3} \mathrm{~N}_{4}$ underlayers on $\mathrm{Si}$ with thicknesses of $10 \mathrm{~nm}$ (red lines) and $30 \mathrm{~nm}$ (blue lines). The cylinders have a diameter and height of $100 \mathrm{~nm}$ and have a $30 \mathrm{~nm} \mathrm{Si}{ }_{3} \mathrm{~N}_{4}$ overcoating.

also the case for a point dipole. ${ }^{8}$ The reduced cross section is due to the fact that the field driving the dipole consists of contributions from the partially reflected scattered field as well as the incident field. As the distance to the interface decreases, interference between the incident and partially reflected scattered field leads to a significant reduction in the driving field. In an optimized light trapping geometry, where the required particle coverage is determined by the scattering cross section, the effect of the dielectric spacer layer must thus be taken into account, in addition to particle size and shape.

In summary, in this paper we have shown that particle shape is a crucial parameter for designing plasmon enhanced solar cells and that $\mathrm{Ag}$ particles with a large fraction of their volume close to the substrate lead to the most effective path length enhancement due to enhanced near-field coupling. Further we have shown that the scattering cross section of the particles can be manipulated by varying the distance of the particles from the substrate. Future work will focus on engineering optimized metal particle arrays, also taking into account interparticle coupling.

This work is part of the Joint Solar Programme (JSP) of FOM, which is financially supported by NWO. The JSP is cofinanced by the Foundation Shell Research.

${ }^{1}$ M. A. Green, Solar cells: Operating Principles, Technology and System Applications (The University of New South Wales, Sydney, 1998).

${ }^{2}$ S. Pillai, K. R. Catchpole, T. Trupke, and M. A. Green, J. Appl. Phys. 101, 093105 (2007).

${ }^{3}$ D. M. Schaadt, B. Feng, and E. T. Yu, Appl. Phys. Lett. 86, 063106 (2005).

${ }^{4}$ C. Hägglund, M. Zäch, G. Petersson, and B. Kasemo, Appl. Phys. Lett. 92, 053110 (2008).

${ }^{5}$ H. R. Stuart and D. G. Hall, Appl. Phys. Lett. 69, 2327 (1996).

${ }^{6}$ S. Pillai, K. R. Catchpole, T. Trupke, G. Zhang, J. Zhao, and M. A. Green, Appl. Phys. Lett. 88, 161102 (2006).

${ }^{7}$ FDTD solutions (www.lumerical.com).

${ }^{8}$ J. Mertz, J. Opt. Soc. Am. B 17, 1906 (2000).

${ }^{9}$ E. Yablonovitch, J. Opt. Soc. Am. 72, 899 (1982).

${ }^{10}$ M. A. Green, Silicon Solar Cells: Advanced Principles and Practice (The University of New South Wales, Sydney, 1995).

${ }^{11}$ G. Xu, M. Tazawa, P. Jin, S. Nakao, and K. Yoshimura, Appl. Phys. Lett. 82, 3811 (2003). 